\title{
Rehabilitation and Neurologic Repair in Parkinson's Disease
}

\author{
Ronald T. Seel ${ }^{\mathrm{a}}$ and David X. Cifu ${ }^{\mathrm{b}}$ \\ ${ }^{a}$ Crawford Research Institute, Shepherd Center, 2020 Peachtree Road, NW, Atlanta, GA 30309, USA \\ Tel.: +1 404367 1240; Fax: +1 404350 3081; E-mail: ron seal@shepherd.org \\ ${ }^{\mathrm{b}}$ Virginia Commonwealth University, Box 980661, 1223 East Marshall Street, Richmond, VA 23298-0661, USA \\ E-mail:dcifu@hsc.vcu.edu
}

\begin{abstract}
Idiopathic Parkinson's disease (PD) is a neurodegenerative disorder and the most common cause of the Parkinsonian syndrome: asymmetric bradykinesia, hypokinesia, and rigidity, sometimes combined with rest tremor and postural changes. Historically, PD treatment has primarily aimed to replace the loss of dopamine through pharmaceutical intervention, which may reduce physical symptoms and slow progression but rarely achieves complete control. More recently, multi-disciplinary neurorehabilitation treatments have been proposed to treat PD sequelae that reduce functional independence and lead to disability. This special issue explores current and future approaches to neurorehabilitation in PD. Papers were contributed in the areas of physical rehabilitation, neuropsychology, speech, deep brain stimulation and stem cell research.

The Veteran's Health Administration (VHA) Parkinson's disease Research, Education, and Clinical Centers (PADRECC) have authored the body of work in this issue. The VHA system competitively awarded these regional centers of PD excellence to improve long-term functional outcomes for veterans. The PADRECC centers partner with affiliated academic medical centers to leverage expertise and resources in order to provide state-of the art treatments, deliver best medical practice education, and conduct innovative research. Diaz and Bronstein provide an overview of this landmark program including program mission and objectives, multidisciplinary services offered, the VHA and National Institute on Neurological Disorders and Stroke (NINDS)
\end{abstract}

co-funded deep brain stimulation (DBS) surgical trial, the identification of PD quality care standards, and the research and development of new therapies including cell replacement. In a second study, Carne et al. examine the efficacy of PADRECC multidisciplinary treatment which includes visits with neurologists, physiatrists, and psychologists as well as functional diagnostic testing, rehabilitation therapies, home exercise programs, support groups, and disease and wellness education. Preliminary evidence from this single center study suggests that almost $70 \%$ of patients showed functional motor improvements during their first year of PADRECC multidisciplinary treatment.

In patients with $\mathrm{PD}$, the incidence of falls is greater than in the age-matched general population, affecting more than $68 \%$ of patients [1]. Seventy percent of falls in PD patients are due to intrinsic factors, and fear of falls is common, often leading to restricted ADLs [2]. Two papers in this issue are devoted to reducing falls. Robinson et al. identify risk factors for falling in PD appropriately grounded within the context of falling in the elderly. Pilot data are presented for tertiary interventions that might reduce specific risk factors. Practical treatment recommendations to reduce specific falling risk factors are proposed based on scientific review and best medical practice. Protas et al. conducted a randomized controlled trial to demonstrate the efficacy of gait training using supported treadmill for individuals with PD. Three hourly sessions over the course of 8 weeks produced significant reduction in falls, improved 
gait speed, and increased stride length compared to controls.

The Goldman Institute on Aging has reported that PD related cognitive and psychological problems are predictive of disability even after controlling for motor dysfunction [3]. However, comprehensive cognitive and psychological testing is often not reimbursed by insurers and can be burdensome to patients and their families. Ong et al. present a brief neuropsychological protocol for the assessment of cognitive and emotional functioning of patients with idiopathic PD. The protocol yielded findings consistent with other PD cognitive and psychological outcome studies. Ecological uses of the brief neuropsychological protocol are detailed.

Speech impairment is an under appreciated sequel of PD that can adversely impact interpersonal relationships and life quality. Trail et al. present a comprehensive literature review on speech disorders and treatments for persons with PD. The authors note that while $89 \%$ of patients with PD have a speech or voice disorder, research suggests that only 3-4\% receive speech treatments. Various treatment techniques are described and evaluated with the Lee Silverman Voice Treatment (LSVT) recommended as best medical practice.

One of the most important PD treatment developments over the last decade has been the use of DBS for persons whose symptoms can no longer be controlled by medication. Piper et al. provide an overview of DBS surgical approaches and their impacts on gait functioning. PADRECC clinical research data are reported that indicate unilateral and bilateral DBS improve parkinsonian gait similar to dopamine replacement through medication. Analyses also indicate that improvements in gait dynamics are sustained for at least 3 to 4 years post-DBS surgery.

The special issue ends with an update on stem cell replacement as a potential future treatment for Parkinson's disease. Fillmore et al. provide progress and challenges to developing cell replacement PD therapies including the identification of optimal cell source, cell growth and differentiation requirements, and target delivery locations. The authors then present a current working model of cell replacement therapy in which patients would be surgically infused with their own stem cells using a specially designed catheter.

In summary, this special issue addresses the importance of neurorehabilitation as a primary treatment modality for the management of PD. The study of neurorehabilitation for $\mathrm{PD}$ is still in its infancy as are the PADRECCs, thus, many of the results in this issue should be regarded as preliminary. Conducting clinical research in an aging population with degenerative illness such as PD will continue to present significant complexity and challenges in our attempts to identify the most efficacious treatments. Even for novel and promising PD treatments such as stem cell replacement therapy, neurorehabilitation will remain essential for patients to regain functioning, providing our rehabilitation therapists with even greater challenges.

\section{References}

[1] B.H. Wood, J.A. Bilclough, A. Bowron and R.W. Walker, Incidence and prediction of falls in Parkinson's disease: a prospective multidisciplinary study, J Neurol Neurosurg Psychiatry 72(6) (Jun 2002), 721-725.

[2] B.R. Bloem, Y.A. Grimbergen, M. Cramer, M. Willemsen and A.H. Zwinderman, Prospective assessment of falls in Parkinson's disease, J Neurol 248(11) (Nov 2001), 950-958.

[3] M. Brod, G.A. Mendelsohn and B. Roberts, Patients' experiences of Parkinson's Disease, Journal of Gerontology 53B(4) (1998), 213-222. 